\title{
Three Weeks Carboplatin/Paclitaxel versus Weekly Regimen in Egyptian Women Cohort Treated for Ovarian Carcinoma
}

\author{
A. Mounir ${ }^{1 *}$, E. E. Ibrahim² \\ ${ }^{1}$ Department of Clinical Oncology, Cairo University, Cairo, Egypt \\ ${ }^{2}$ Department of Radiation Oncology, International Medical Center (IMC), Cairo, Egypt \\ Email: *achrafmounir@hotmail.com
}

How to cite this paper: Mounir, A. and Ibrahim, E.E. (2021) Three Weeks Carboplatin/Paclitaxel versus Weekly Regimen in Egyptian Women Cohort Treated for Ovarian Carcinoma. Journal of Cancer Therapy, 12, 78-85.

https://doi.org/10.4236/jct.2021.122009

Received: January 17, 2021

Accepted: February 16, 2021

Published: February 19, 2021

Copyright (๑) 2021 by author(s) and Scientific Research Publishing Inc. This work is licensed under the Creative Commons Attribution International License (CC BY 4.0).

http://creativecommons.org/licenses/by/4.0/

\section{(c) (i) Open Access}

\begin{abstract}
Introduction: Epithelial Ovarian Carcinoma (EOC) comprises the vast majority (almost 90\%) of ovarian carcinomas. Chemotherapy is the main treatment in ovarian cancers. The standard of care in the chemotherapeutic is the combination of a platinum (carboplatin or cisplatin) and a taxane (paclitaxel or docetaxel). Studies were done to determine whether this combination to be given weekly or every 3 weeks. Patient and Method: Inclusion criteria: 1) Female patients between the ages of $17-78$ years. 2) Baseline hematological, renal and liver laboratory profiles were within accepted ranges. 3) Performance status of the patients was 0-II. 4) Patients were pathologically proven ovarian cancer. 5) A follow-up period for at least 6 months was required. Exclusion criteria: 1) Patients who had double malignancy were excluded. 2) Performance status more than II. 3) Other comorbidity. Results: We reviewed 69 female patients with EOC, with $60 \%$ received every three weeks regimen. Mean age was 53.22 years. At a median follow up of 45.9 months, there was no significant different between the two protocols in terms of mean PFS, 62.35 months (95\% CI: 50.08 - 74.63 months) for the three-weekly cohort, and 69.25 months (95\% CI: 55.24 - 83.26 months) for weekly protocol $(\mathrm{p}=0.613)$. The three weekly regimen patients had a higher incidence of hospital admission ( $40 \%$ vs $18.5 \%$ for the weekly protocol patients), but it didn't reach a statistical significance $(\mathrm{p}=0.063)$. The three weekly protocol had a significantly higher incidence of causing a neutropenic fever $(p=0.003)$. Conclusion: In our cohort of Egyptian women with EOC, no significant difference in PFS was found when compared the weekly Carboplatin/paclitaxel when compared to the classic three weeks, although the weekly protocol may be causing less febrile neutropenia and fewer hospital admissions.
\end{abstract}




\section{Keywords}

Epithelial Ovarian Carcinoma, Carboplatin/Paclitaxel, Weekly versus Every Three Weeks

\section{Introduction}

Ovarian cancer is classified in various categories, with the Epithelial Ovarian Carcinoma (EOC) comprising the vast majority (almost 90\%). Although it is not the most common gynecological cancer, EOC keeps to be the most lethal. Cancer statistics registry in the United States showed that the estimated cases were 21,750 , with estimated deaths of 13,940 [1]. EOC typically presents at late stage, and more than $>80 \%$ are diagnosed with advanced stage [2].

Chemotherapy is the main treatment in ovarian cancers, given in most stages of the disease either as neoadjuvant or adjuvant or as a palliative treatment. The standard of care in the chemotherapeutic management of ovarian cancer is the combination of a platinum (carboplatin or cisplatin) and a taxane (paclitaxel or docetaxel) given intravenously. Studies were done to determine whether the regimen Carboplatin and paclitaxel given once a week or the standard regimen administered every 3 weeks, would have a better outcome in terms of PFS and the toxicity profile. The NRG-GOG study, by Chan et al., compared the regimen Carboplatin and paclitaxel given once a week compared with the standard regimen administered every 3 weeks regards the side effects, PFS and toxicity profile. After a median follow-up of 28 months, weekly paclitaxel did not appreciably prolong progression-free survival, as compared with paclitaxel administered every 3 weeks $(p=0.18)$. Although the two protocols had a different toxicity profiling e.g., anemia was more common in the weekly protocol, while neutropenia was more common in every three weeks regimen [3].

In this study, we reviewed a cohort of Egyptian women received either protocols and reported on the different PFS and toxicity patterns. This retrospective study was carried out in Kasr Al Ainy center of oncology and nuclear medicine (NEMROCK), Faculty of Medicine, Cairo University. We revised the files of patients with EOC presented to NEMROCK in a period starting from January 2011 to December 2015.

\section{Results}

\subsection{Patient, Disease and Treatment}

Among the reviewed patient files, only 69 female patients had a complete data set for analysis. Mean age was 53.22 years (Range: 22 - 74). Sociodemographic data are listed in Table 1. Disease and treatment data are listed in Table 2.

\subsection{Progression Free Survival (PFS)}

At a median follow up of 45.9 months, the median PFS of the cohort wasn't 
reached, but the mean was 66.42 months (Standard Deviation $\pm 4.8,95 \%$ CI: 56.93 - 75.91 months) (Figure 1).

PFS was significantly affected by the first line of treatment received and the extent of the surgical resection as follow:

First line of treatment:

Data showed that the mean of PFS was 52.6 months for patients who received chemotherapy as $1^{\text {st }}$ line of treatment and 71.6 months for the patients who underwent surgery as $1^{\text {st }}$ line $(p=0.087)$ (Figure 2$)$.

Extent of surgical resection:

Data showed that the mean of PFS was 74.8 months for patients who underwent optimal surgery, 64.6 months for those who underwent sub-optimal surgery and 60.2 months for patients who didn't undergo surgery $(\mathrm{p}=0.130)$ (Figure 3 ).

Forty patients $(60 \%)$ received three weeks protocol and $27(40 \%)$ received the weekly protocol. There was no significant different between the two protocols in terms of PFS $(\mathrm{p}=0.613)$. For the three-weekly protocol, $13 / 40(32 \%)$ patients had progressed, while progression events had occurred in $8 / 27$ (30\%). For the three-weekly cohort, the mean PFS was 62.35 months (Standard Deviation \pm 6.26, 95\% CI: 50.08 - 74.63 months), while for the weekly protocol was 69.25 months (Standard Deviation \pm 7.15 , 95\% CI: 55.24 - 83.26 months) (Figure 4).

Table 1. Sociodemographic data.

\begin{tabular}{ccc}
\hline & $<20$ ys & $0 \%$ \\
Age & $21-40$ ys & $15 \%$ \\
& $40-60 \mathrm{~s}$ & $55 \%$ \\
\hline Geographic distribution & $>60$ ys & $30 \%$ \\
\hline Marital status & Cairo & $27 \%$ \\
& Upper Egypt & $30 \%$ \\
\hline
\end{tabular}

Table 2. Disease and treatment criteria.

\begin{tabular}{ccc}
\hline \multicolumn{2}{c}{ Endothelial Ovarian Carcinoma } & Number (\%) \\
\hline & Serous & $54(80 \%)$ \\
Histopathological Type & Endometroid & $5(71 \%)$ \\
& Mucinous & $6(81 \%)$ \\
& Clear Cell & $2(21 \%)$ \\
\hline \multirow{2}{*}{ Initial Treatment } & Chemotherapy & $24(36 \%)$ \\
& Surgery & $43(64 \%)$ \\
\hline \multirow{2}{*}{ Regimen of Adjuvant } & Carboplatin/Paclitaxel every 3 weeks & $40(60 \%)$ \\
& Carboplatin/Paclitaxel weekly & $27(40 \%)$
\end{tabular}




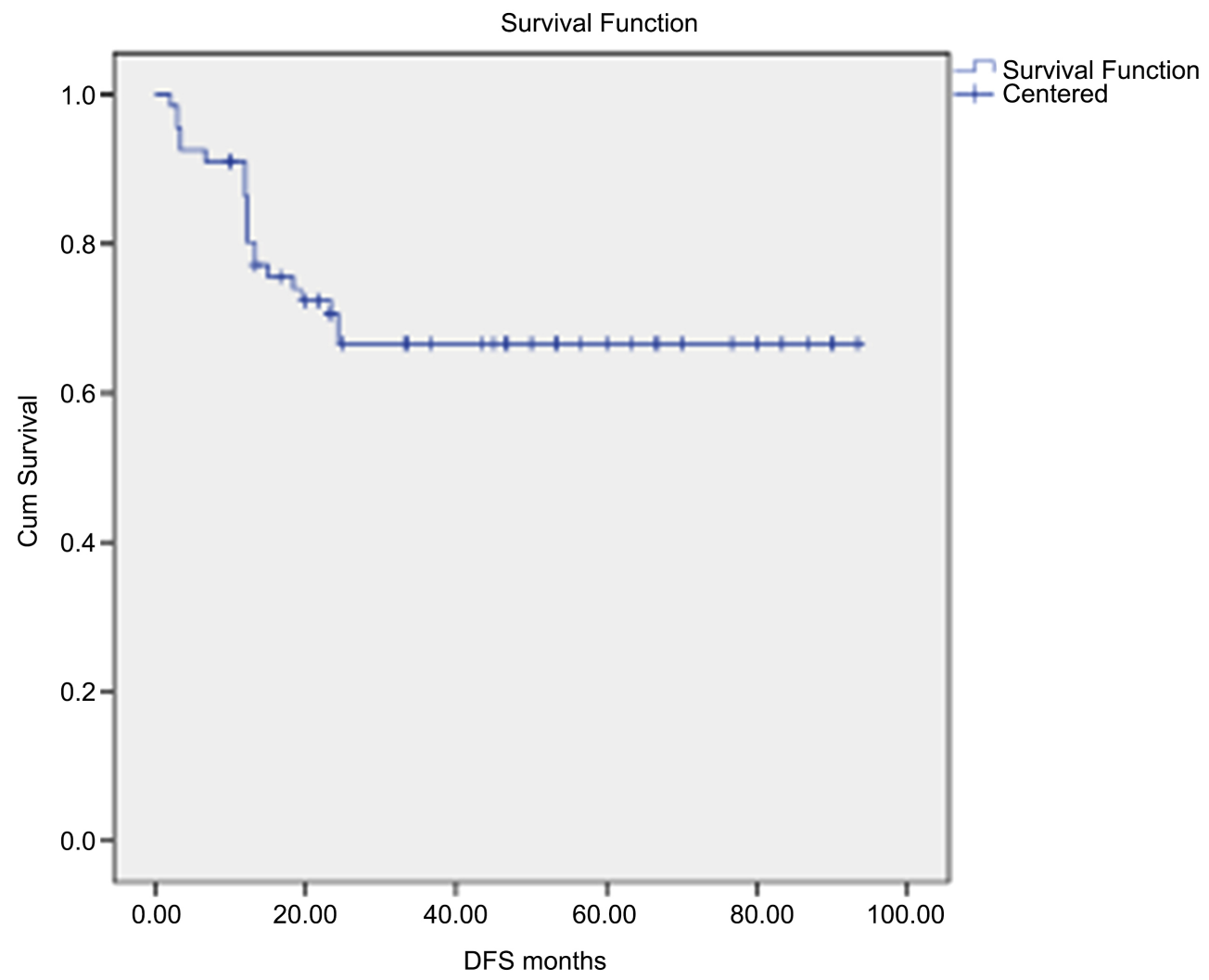

Figure 1. Progression Free Survival (PFS).

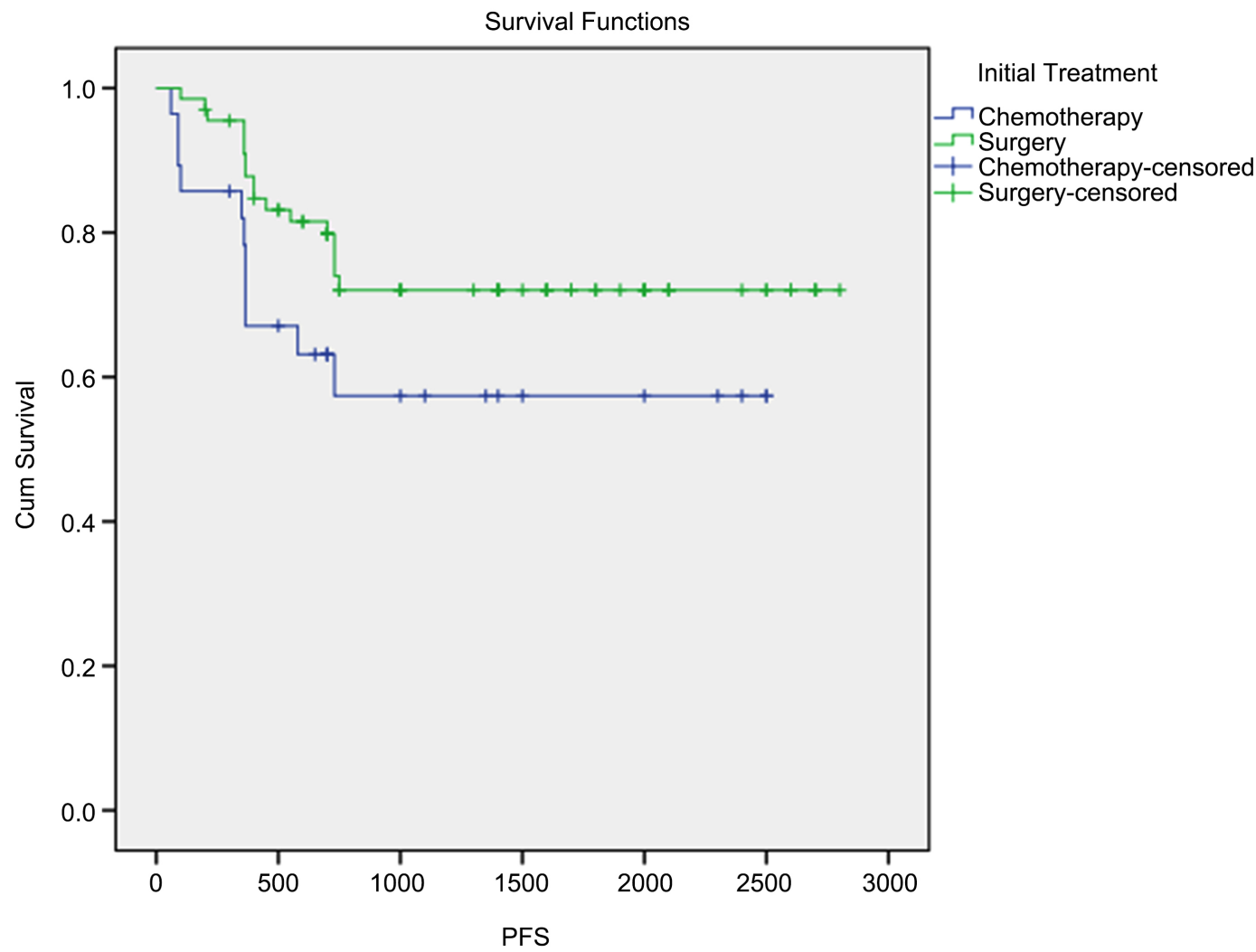

Figure 2. PFS according to $1^{\text {st }}$ line of ttt. 


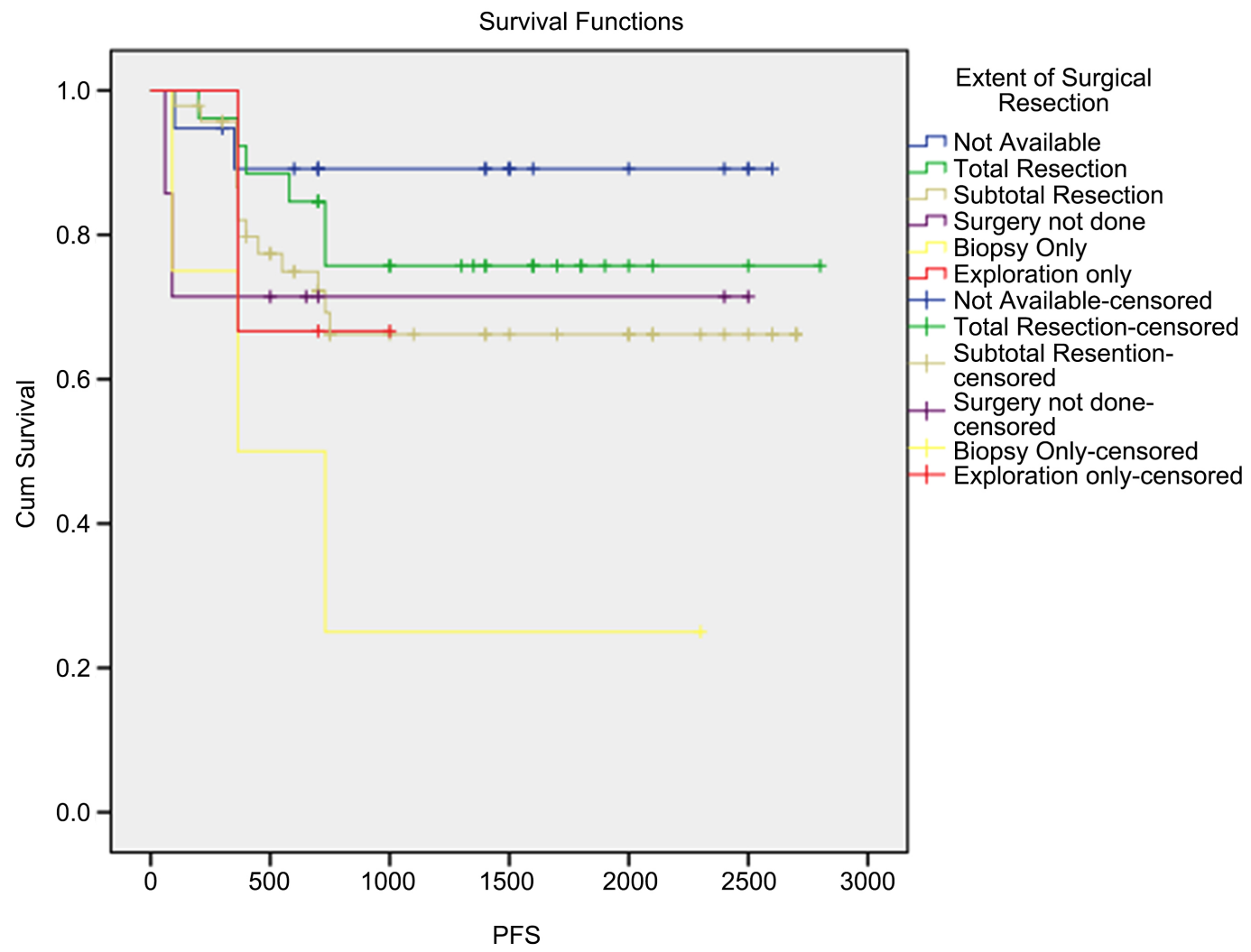

Figure 3. PFS according to extent of surgery.

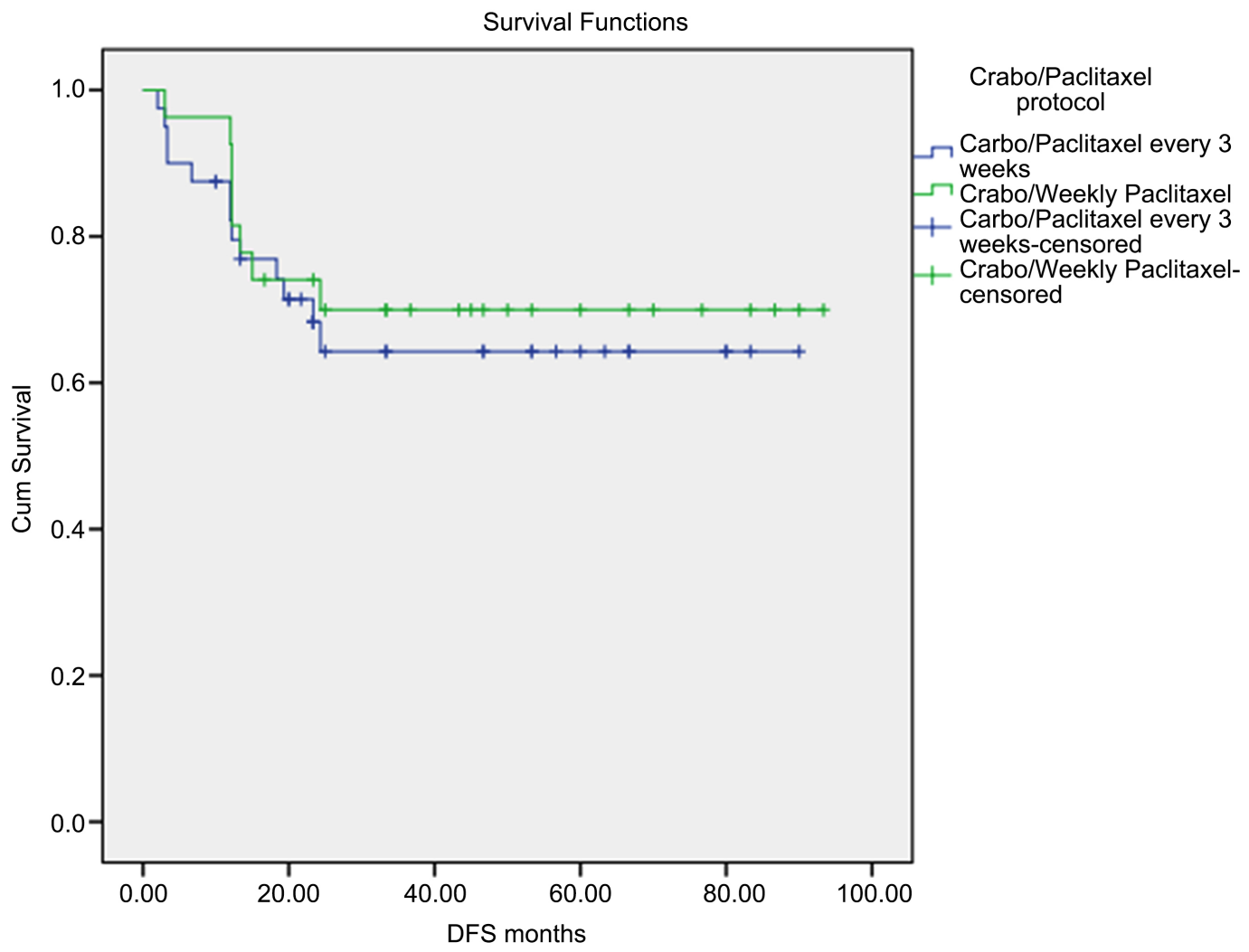

Figure 4. PFS: Weekly versus three weeks Carboplatin/paclitaxel. 


\subsection{Complications and Toxicity Profile Comparison}

We reported on the incidence of hospital admission and the neutropenic fever in both groups. For the hospital admission, the three weekly regimen patients had a higher incidence of being admitted (40\% vs $18.5 \%$ for the weekly protocol patients), but it didn't reach a statistical significance $(p=0.063)$. The three weekly protocol had a significantly higher incidence of causing a neutropenic fever ( $\mathrm{p}=$ $0.003)$. Only 5 patients $(18.5 \%)$ of the weekly regimen developed neutropenic fever, versus 22 patients (55\%) of the three-weekly regimen (Table 3 ).

\section{Discussion}

Starting from the year 2010, there was a debate about the potential superiority of using dose dense protocols in ovarian cancer e.g., weekly Carboplatin/paclitaxel versus every three weeks protocol. Between the years of 2011 and 2014, there was four pivotal studies running, aiming to answer that question: The NRG/GOG-0262 [3], ICON-8 [4], MITO-7 studies [5] and the JGOG 3016 trial [6].

In our study, we retrospectively revised the female patients treated at NEMROCK at a parallel period of time of debate e.g., January 2011 to December 2015. We found that $60 \%$ of the received the classic every three weeks protocol at that time, versus $40 \%$ patients received the challenging weekly protocol. At a median follow up of 45.9 months, there was no significant difference in the mean PFS between the two protocols: 62.35 months for every three weeks (Standard Deviation \pm 6.26 , 95\% CI: 50.08 - 74.63 months), while for the weekly protocol was 69.25 months (Standard Deviation $\pm 7.15,95 \%$ CI: $55.24-83.26$ months) ( $\mathrm{p}=$ $0.613)$.

Such finding coincides with the finding of the NRG study PFS was 14.7 months for weekly protocol versus 14.0 months for the three weeks regimen, hazard ratio for disease progression or death, 0.89 (95\% confidence interval [CI], 0.74 to 1.06; $\mathrm{p}=0.18)$, and the MITO-7 PFS which was found to be (17.3 months: $95 \%$ CI 15.2 - 20.2) in patients assigned to treatment every 3 weeks, versus 18.3 months (16.8 - 20.9) in women allocated to the weekly schedule (hazard ratio $0.96,95 \%$

Table 3. Toxicity Profile: Weekly versus three weeks Carboplatin/paclitaxel.

\begin{tabular}{cccccc}
\hline \multirow{2}{*}{ Carboplatin/Paclitaxel every 3 weeks } & & No & 24 & $60.0 \%$ & \\
& $\begin{array}{c}\text { Hospital } \\
\text { Admission }\end{array}$ & Yes & 16 & $40.0 \%$ & \\
Carboplatin/Paclitaxel weekly & & No & 22 & $81.5 \%$ & \\
& & Yes & 5 & $18.5 \%$ & \\
Carboplatin/Paclitaxel every 3 weeks & & No & 18 & $45.0 \%$ & \\
& Neutropenic & Yes & 22 & $55.0 \%$ & \\
Fever & No & 22 & $81.5 \%$ & $\mathrm{p}=0.003$ \\
Carboplatin/Paclitaxel weekly & & Yes & 5 & $18.5 \%$ & \\
\hline
\end{tabular}


CI $0.80-1.16 ; p=0.66$ ) [3] [5]. The ICON-8 study randomized the EOC patients into three groups (two dose-dense weekly regimens and the standard 3-weekly chemotherapy). Group 1 received (carboplatin area under the curve [AUC] 5 or AUC6 and $175 \mathrm{mg} / \mathrm{m}^{2}$ paclitaxel every 3 weeks), group 2 received (carboplatin AUC5 or AUC6 every 3 weeks and $80 \mathrm{mg} / \mathrm{m}^{2}$ paclitaxel weekly), and group 3 received (carboplatin AUC2 and $80 \mathrm{mg} / \mathrm{m}^{2}$ paclitaxel weekly). Again, no significant improvement was found when compared the three arms: mean survival time was 24.4 months [97.5\% CI 23.0 - 26.0] in group 1, 24.9 months [24.0 25.9] in group 2, and 25.3 months [23.9 - 26.9] in group 3. The median progression-free survival was 17.7 months [IQR 10.6-not reached] in group 1, 20.8 months [11.9 - 59.0] in group 2, 21.0 months [12.0 - 54.0] in group 3 ( $p=0.35$ for group 2 vs group 1; group 3 vs $1 \mathrm{p}=0.51$ ) [4].

These agreement of no prolongation of PFS by the dose dense protocols is challenged by the Japanese study results (JGOG 3016), which found that Median PFs was significantly higher in the weekly protocol (28.2 months [95\% CI 22.3 33.8], versus the classic three weeks treatments (17.5 months [15.7 - 21.7]) hazard ratio [HR] 0.76, 95\% CI 0.62 - 0.91; ( $p=0.0037)$ [6].

The JGOG protocol had the advantage of the longer median follow up among the studies i.e., 76.8 months compared to 28 months of the NRG study, and 22.3 months in the MITO-7 study. Although in our study, with an intermediate median follow up of 45.9 months, we didn't find that significant PFS difference that was concluded in JGOG 3016 trial [3] [5] [6].

For the hospital admission, the three weekly regimen patients had a higher incidence of being admitted (40\% vs $18.5 \%$ for the weekly protocol patients), although it was hardly touching the level of statistical significance $(p=0.063)$. As regards to febrile neutropenia, the three weekly protocol had a significantly higher incidence of causing a neutropenic fever $(p=0.003)$. Similar results were conducted by the Sparano, et al. study, which showed a lower rate of neutropenic fever in groups receiving weekly paclitaxel than in those receiving paclitaxel every 3 weeks [7]. Also, the NRG study showed that neutropenia occurred less often in the group that received weekly paclitaxel than in the group that received paclitaxel every 3 weeks ( $72 \%$ [246 of 340 patients] vs. $83 \%$ [286 of 343], p < $0.001)[3]$.

\section{Conclusion}

In our cohort of Egyptian women with EOC, no significant difference in PFS was found when compared the weekly Carboplatin/paclitaxel when compared to the classic three weeks, although the weekly protocol may be causing less febrile neutropenia and fewer hospital admissions.

\section{Conflicts of Interest}

The authors declare no conflicts of interest regarding the publication of this paper. 


\section{References}

[1] Siegel, R.L., Miller, K.D. and Jemal, A. (2020) Cancer Statistics. CA: A Cancer Journal for Clinicians, 70, 7-30. https://doi.org/10.3322/caac.21590

[2] National Cancer Institute. Cancer Stat Facts: Ovarian Cancer. https://seer.cancer.gov/statfacts/html/ovary.html

[3] Chan, J.K., Brady, M.F., Penson, R.T., Huang, H., Birrer, M.J., Walker, J.L., DiSilvestro, P.A., Rubin, S.C., Martin, L.P., Davidson, S.A., Huh, W.K., O’Malley, D.M., Boente, M.P., Michael, H. and Monk, B.J. (2016) Weekly vs. Every-3-Week Paclitaxel and Carboplatin for Ovarian Cancer. The New England Journal of Medicine, 374, 738-748. https://doi.org/10.1056/NEJMoa1505067

[4] Clamp, A.R., James, E.C., McNeish, I.A., Dean, A., Kim, J.W., O’Donnell, D.M., Hook, J., Coyle, C., Blagden, S., Brenton, J.D., Naik, R., Perren, T., Sundar, S., Cook, A.D., Gopalakrishnan, G.S., Gabra, H., Lord, R., Dark, G., Earl, H.M., Hall, M., Banerjee, S., Glasspool, R.M., Jones, R., Williams, S., Swart, A.M., Stenning, S., Parmar, M., Kaplan, R. and Ledermann, J.A. (2019) Weekly Dose-Dense Chemotherapy in First-Line Epithelial Ovarian, Fallopian Tube, or Primary Peritoneal Carcinoma Treatment (ICON8): Primary Progression Free Survival Analysis Results from a GCIG Phase 3 Randomised Controlled Trial. Lancet, 394, 2084-2095.

https://doi.org/10.1016/S0140-6736(19)32259-7

[5] Pignata, S., Scambia, G., Katsaros, D., Gallo, C., Pujade-Lauraine, E., De Placido, S., Bologna, A., Weber, B., Raspagliesi, F., Panici, P.B., Cormio, G., Sorio, R., Cavazzini, M.G., Ferrandina, G., Breda, E., Murgia, V., Sacco, C., Cinieri, S., Salutari, V., Ricci, C., Pisano, C., Greggi, S., Lauria, R., Lorusso, D., Marchetti, C., Selvaggi, L., Signoriello, S., Piccirillo, M.C., Di Maio, M., Perron, F., Multicentre Italian Trials in Ovarian Cancer (MITO-7), Groupe d'Investigateurs Nationaux pour l'Etude des Cancers Ovariens et du sein (GINECO), Mario Negri Gynecologic Oncology (MaNGO), European Network of Gynaecological Oncological Trial Groups (ENGOT-OV-10), Gynecologic Cancer InterGroup (GCIG) Investigators. (2014) Carboplatin plus Paclitaxel Once a Week versus Every 3 Weeks in Patients with Advanced Ovarian Cancer (MITO-7): A Randomised, Multicentre, Open-Label, Phase 3 Trial. The Lancet Oncology, 15, 396-405. https://doi.org/10.1016/S1470-2045(14)70049-X

[6] Katsumata, N., Yasuda, M., Isonishi, S., Takahashi, F., Michimae, H., Kimura, E., Aoki, D., Jobo, T., Kodama, S., Terauchi, F., Sugiyama, T., Ochiai, K., Japanese Gynecologic Oncology Group. (2013) Long-Term Results of Dose-Dense Paclitaxel and Carboplatin Versus Conventional Paclitaxel and Carboplatin for Treatment of Advanced Epithelial Ovarian, Fallopian Tube, or Primary Peritoneal Cancer (JGOG 3016): A Randomised, Controlled, Open-Label Trial. The Lancet Oncology, 14, 1020-1026. https://doi.org/10.1016/S1470-2045(13)70363-2

[7] Sparano, J.A., Wang, M., Martino, S., Jones, V., Perez, E.A. and Saphner, T. (2008) Weekly Paclitaxel in the Adjuvant Treatment of Breast Cancer. New England Journal of Medicine, 358, 1663-1671. https://doi.org/10.1056/NEJMoa0707056 